\title{
Universiteit
}

Leiden

The Netherlands

\section{Method for Probing the Orbital Angular Momentum of Optical Vortices in Electromagnetic Waves from Astronomical Objects}

Berkhout, G.C.G.; Beijersbergen, M.W.

\section{Citation}

Berkhout, G. C. G., \& Beijersbergen, M. W. (2008). Method for Probing the Orbital Angular Momentum of Optical Vortices in Electromagnetic Waves from Astronomical Objects. Physical Review Letters, 101(10), 100801. doi:10.1103/PhysRevLett.101.100801

Version: $\quad$ Not Applicable (or Unknown)

License: $\quad$ Leiden University Non-exclusive license

Downloaded from: https://hdl.handle.net/1887/67462

Note: To cite this publication please use the final published version (if applicable). 


\title{
Method for Probing the Orbital Angular Momentum of Optical Vortices in Electromagnetic Waves from Astronomical Objects
}

\author{
Gregorius C. G. Berkhout ${ }^{1,2, *}$ and Marco W. Beijersbergen ${ }^{1,2}$ \\ ${ }^{1}$ Huygens Laboratory, Leiden University, P.O. Box 9504, 2300 RA Leiden, The Netherlands \\ ${ }^{2}$ cosine Science \& Computing BV, Niels Bohrweg 11, 2333 CA Leiden, The Netherlands
}

(Received 1 July 2008; published 5 September 2008)

\begin{abstract}
We present an efficient method for probing the orbital angular momentum of optical vortices of arbitrary sizes. This method, based on a multipoint interferometer, has its most important application in measuring the orbital angular momentum of light from astronomical sources, opening the way to interesting new astrophysics. We demonstrate its viability by measuring the orbital angular momentum of Laguerre-Gaussian laser beams.
\end{abstract}

DOI: 10.1103/PhysRevLett.101.100801

PACS numbers: 07.60.Ly, 42.50.Tx, 95.85.Kr, 95.55.Cs

It is well understood that light carries angular momentum that, under given circumstances, can be separated into spin and orbital angular momentum [1,2]. The spin angular momentum is associated with polarization and its transfer to a material body has been measured by Beth [3]. The orbital angular momentum (OAM) is associated with an optical vortex and gives rise to a complex field amplitude of the form $\sim \exp (i l \phi)$ and thus a twisted wave front. Such a field has an on-axis singularity resulting in a central dark point in the intensity profile and every photon in it carries an OAM of $l \hbar$ [1]. It has been demonstrated that the OAM of a laser beam enables it to rotate trapped particles [4].

Laser beams with OAM occur spontaneously as higherorder transverse modes or can be created using a spiral phase plate (SPP) [5] or a fork hologram (FH) [6,7]. Optical vortices also occur in speckle patterns $[8,9]$, where until now only vortices with topological charge -1 and +1 [10] have been observed and higher-order zeros are, although not strictly forbidden, very unlikely.

Recently, the interest for OAM of light in astrophysics has grown [11]. Several possible sources of OAM have been suggested, from bright point sources behind a turbulent interstellar medium to the cosmic microwave background (CMB). Measurement of the OAM of the associated vortices could open the way to interesting new astrophysics. Due to the large propagation distances one expects the intensity variation of astronomical optical vortices to be on very large scales.

Determining the OAM state of an optical vortex requires knowledge of the phase distribution around the singularity. Thidé et al. proposed a method for generating and detecting OAM in low-frequency radio beams [12], using an antenna array for coherent measurement of the local field vector in a finite number of points and software to reconstruct the incident vector field. We propose a method based on measuring only the phase instead of the full electric vector, that would allow detection of OAM at optical wavelengths.
Since direct measurement of the phase in the visible regime is not possible, one needs to rely on interferometric techniques. Leach et al. [13] proposed an interferometric method for measuring the OAM of a single photon. A more commonly used technique is to interfere the wave front under study with a flat wave front $[14,15]$, in which case the interferogram reveals the OAM state of the optical vortices. This technique can also be applied to less symmetric optical vortices such as those occurring in speckle patterns, where the interferogram shows their position, OAM state and skewness [8]. In principle, this method allows one to distinguish between infinitely many states.

When the optical vortex is large compared to the detector area, it becomes difficult to measure the phase distribution, since the dark region around the singularity is accordingly larger, leaving less light to interfere with. Moving the detector away from the axis of the vortex towards areas of higher intensity does not solve this, since the amount of phase change over a given area decreases as one moves radially outwards. A possible solution is to use more that one aperture roughly separated by the size of the optical vortex. Performing Young's double slit experiment [16] with Laguerre-Gaussian beams indeed reveals information about the OAM state of the beam [17]. However in this case the length of the slits has to be of the order of the size of the beam, which in many astronomical cases is infeasible.

This problem can be solved by using pointlike apertures instead of slits. In this Letter we describe a system of two or more points, enclosing the singularity of the optical vortex, which we call a multipoint interferometer (MPI), and show that it can be used to measure the OAM state of an optical vortex. We experimentally realize this system by replacing the points with pinholes. This so-called multipinhole interferometer has been studied in the context of partially coherent light $[18,19]$ where the resulting interference patterns reveals information about the coherence properties of the illuminating beam. 
We start by studying the proposed method theoretically. The most convenient class of optical vortices are the Laguerre-Gaussian (LG) beams, which have a complex field amplitude given by

$$
u_{p l}(r, \phi, z) \propto r^{l} L_{p}^{l}\left(\frac{2 r^{2}}{w^{2}}\right) \exp \left(-\frac{r^{2}}{w^{2}}\right) \exp (-i l \phi),
$$

where $w$ is the waist size of the beam, $L_{p}^{l}\left(2 r^{2} / w^{2}\right)$ is the associated Laguerre polynomial, $p$ is the radial mode index and $l$ is the azimuthal mode index.

Since the optical vortices are rotationally symmetric in intensity, we choose to uniformly distribute the points in the MPI in a circle, centered around the singularity of the optical vortex. A general MPI consists of $N$ points, has radius $a$ and lies in the $x y$ plane (see Fig. 1).

Since we consider the Fraunhofer limit, the far-field intensity pattern $I_{l}^{N}$ behind a general MPI illuminated by an on-axis, normally incident LG beam is given by the Fourier transform of the field distribution in the aperture plane

$$
\begin{aligned}
I_{l}^{N}(x, y, z) \propto & \mid \sum_{n=0}^{N-1} \exp \left(-i l \alpha_{n}\right) \\
& \times\left.\exp \left(i \frac{k a}{z}\left(x \cos \alpha_{n}+y \sin \alpha_{n}\right)\right)\right|^{2}
\end{aligned}
$$

where $k=2 \pi / \lambda$ is the wave number and $\alpha_{n}=2 \pi n / N$ the azimuthal coordinate of the $n$th point. For two pinholes and a Gaussian beam (i.e., $l=0$ ), Eq. (2) reduces to $I_{0}^{2} \propto$ $\cos ^{2}(\mathrm{kax} / \mathrm{z})$, reproducing the result for the central part of the interference pattern in Young's experiment with two pinholes. If the number of points goes to infinity, corresponding to an annular aperture, Eq. (2) converges to the Bessel function of the first kind of order $|l|, J_{|l|}(\mathrm{kar} / \mathrm{z})$, where $r$ is the radial distance. For other values of $N$ and $l$,

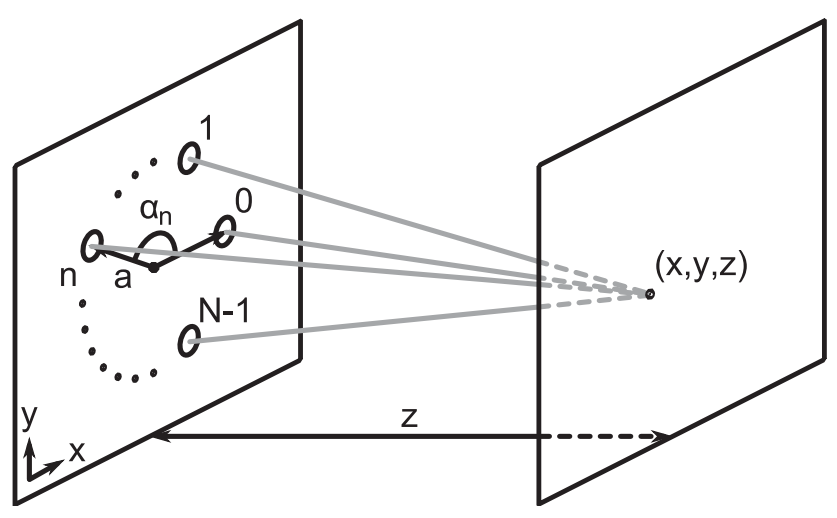

FIG. 1. Geometry and notation of a generic multipoint interferometer consisting of $N$ points, uniformly distributed over a circle of radius $a$ in the $x y$ plane. The points are indicated by open dots and the angular coordinate of the $n$th point is $\alpha_{n}=$ $2 \pi n / N$.
Eq. (2) yields unexpectedly complex patterns, some of which are shown in Fig. 2.

A nonzero azimuthal mode index has a significant effect on the observed patterns, as can be seen by comparing the second and third column of Fig. 2 to the first. The results for $N=2$ are easily understood; the azimuthal phase

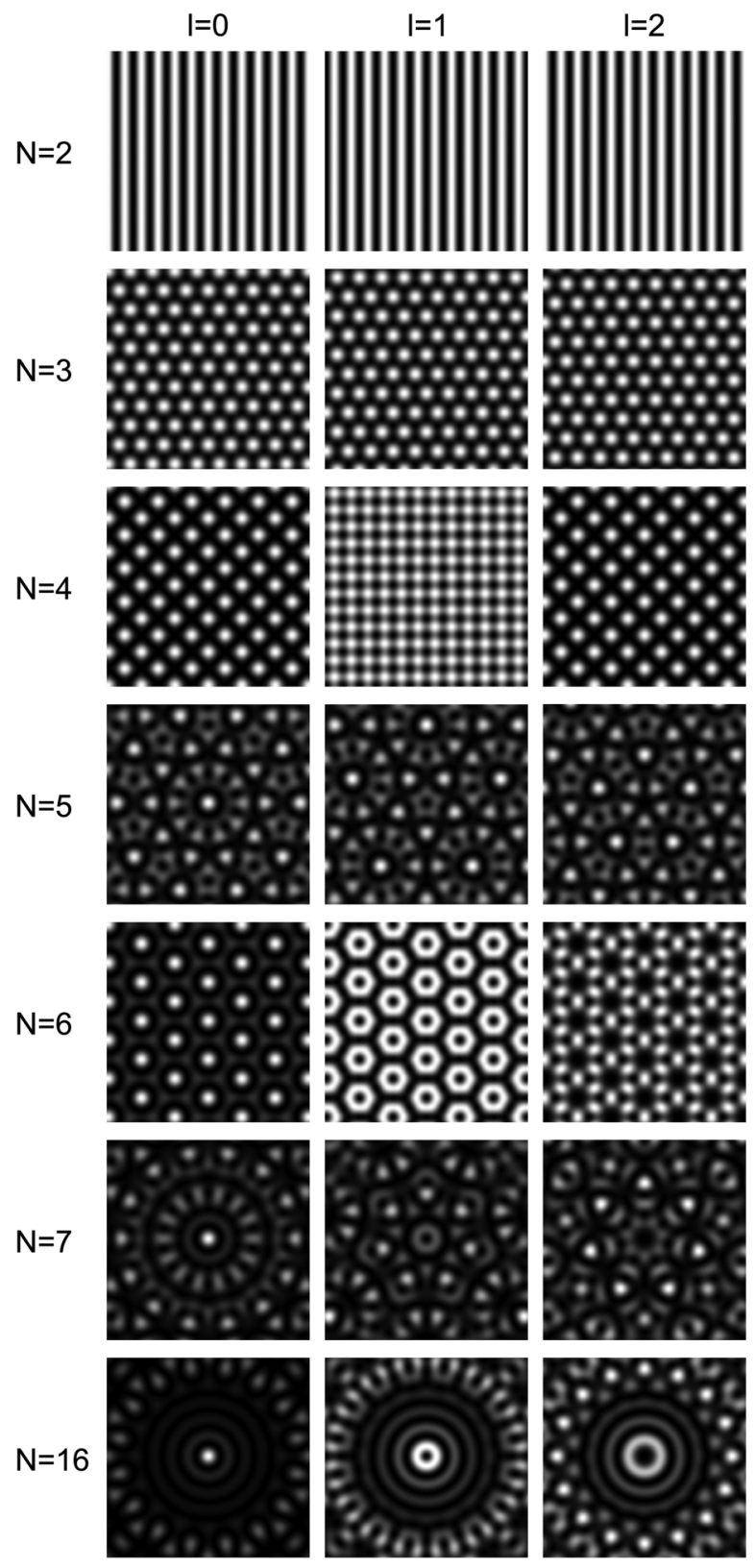

FIG. 2. Far-field intensity patterns behind an MPI of $N$ points illuminated by an LG beam with azimuthal mode index $l$ calculated from Eq. (2) (linear gray scale, white corresponds to high intensity). $N=2, l=0$ reproduces the results for Young's experiment with two pinholes. $N=16$ hints at the fact that the observed patterns converge to a Bessel function of order $|l|$. At intermediate values the pattern is very dependent on the azimuthal mode index $l$, and therefore the OAM of the field at the location of the MPI. 
dependence of the LG beam introduces a phase difference of $\pi$ between the two points for odd $l$, shifting the observed patterns by half a period with respect to the patterns for even $l$. This phase difference can however also be introduced by a tilt of the illuminating beam with respect to the plane of the MPI, making it impossible to distinguish between OAM and a tilt of the beam. The same arguments hold for $N=3$ where we observe three shifted patterns that repeat for $l^{\prime}=l+3$. A two or three point interferometer can therefore only be used to measure the OAM if the optical axis of the illuminating beam is known.

This ambiguity is however removed in the case of four or more points, where the phase distribution caused by different values of $l$ is inherently different from the phase differences introduced by a tilt of the incoming wave front. This can be seen from simple geometry, and it is also evident in the resulting patterns. For four points some $l$ states result in a shift of the pattern but, more importantly, others in qualitatively different patterns. Even though the patterns for five points look similar by eye, all patterns in this case differ significantly, in particular in the details surrounding the bright spots. Further simulations show that for an MPI of $N$ points we observe $N$ different patterns. The patterns for positive and negative $l$ are mirrored in the $x$ axis (see Fig. 3). For an even number of points the patterns are symmetric in the $x$ axis and in this case there is no difference between the pattern for positive and negative $l$, making it impossible to differentiate between negative and positive OAM states, reducing the number of distinguishable states to $N / 2-1$.

Further simulations have shown that the observed qualitative patterns change only marginally when the phase front is not perpendicular to the interferometer plane, proving that an MPI is an efficient OAM detector if it consists of at least four points.

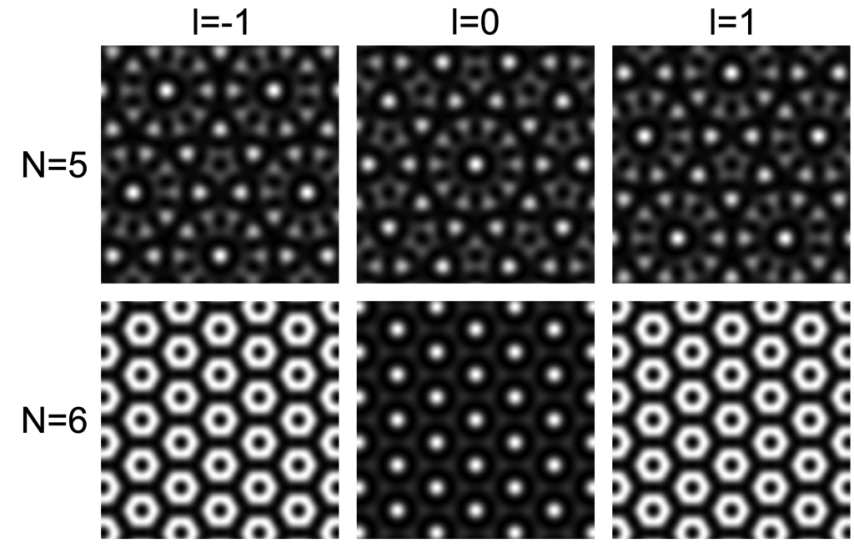

FIG. 3. Simulated far-field intensity patterns behind an MPI of $N$ points illuminated by an LG beam with azimuthal mode index $l$ calculated from Eq. (2). The patterns for $l$ values of opposite sign are mirrored in the $x$ axis. For an odd number of points one can determine the sign of $l$.
To demonstrate the method we measured the interference patterns behind a multi-pinhole interferometer, where the diffraction of the light on the pinholes is used to overlap the light from the different points. We assume that the finite size of the pinholes does not change the interference pattern qualitatively, but adds a convolution with the diffraction pattern of a single pinhole to it. The size of the multi-pinhole interferometer is chosen to be of the order of the waist size of the beam in order to collect maximum intensity from the donut-shaped intensity profile of an $l=1$ laser beam.

We built a setup to study the interference patterns (see Fig. 4 for details) and measured the interference pattern for the different multi-pinhole interferometers for $l$ between -3 and +3 . All observed patterns show excellent agreement with the simulations as can be seen by comparing Figs. 2 and 5. The effect of the finite size of the pinholes can be seen at the edges of the patterns where the intensity drops.

Even if the LG beam is not perfectly on-axis and perpendicular, the observed pattern can still be distinguished clearly, as long as the singularity is located within the circle formed by the pinholes. For example in Fig. 5, the observed pattern for $N=4, l=1$ is skewed but can still be clearly differentiated from $l=0$ and $l=2$.

We demonstrated that the interference pattern behind an MPI illuminated by an LG beam yields information about the azimuthal mode index $l$ of the beam. To our knowledge this is the first method that can be used to measure the OAM of light, without requiring coverage of a large area of the field. As our detection method relies only on a finite number of point measurements, it can be scaled to arbitrarily large sizes and can thus be used to measure arbitrarily large optical vortices such as expected to come from astronomical sources. In the experiments that we performed, we used the diffraction of light on small pinholes to overlap the light from the different points. With this method, the position of the far field increases with increasing separation of the points, leading to increasingly large system size, and increasingly small periodicity of the patterns, making it more difficult to detect. In order to increase the size of the MPI, extra measures have to be

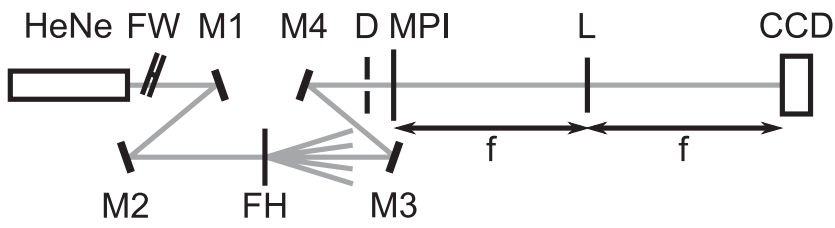

FIG. 4. Setup to measure interference patterns behind a multipinhole interferometer. A helium-neon laser (HeNe), a filter wheel (FW), two mirrors (M1 and M2), and a fork hologram (FH) are used to create LG beams and a set of mirrors (M3 and M4) and a diaphragm (D) are used to select an LG beam to illuminate the multi-pinhole interferometer (MPI). A CCD camera and a lens (L) are used to record the far-field image. 
$\mathrm{I}=0$
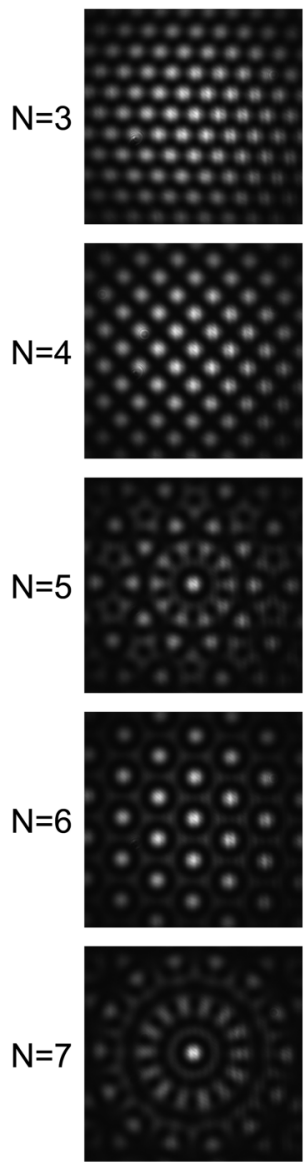

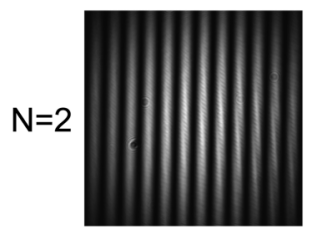

$\mathrm{l}=1$
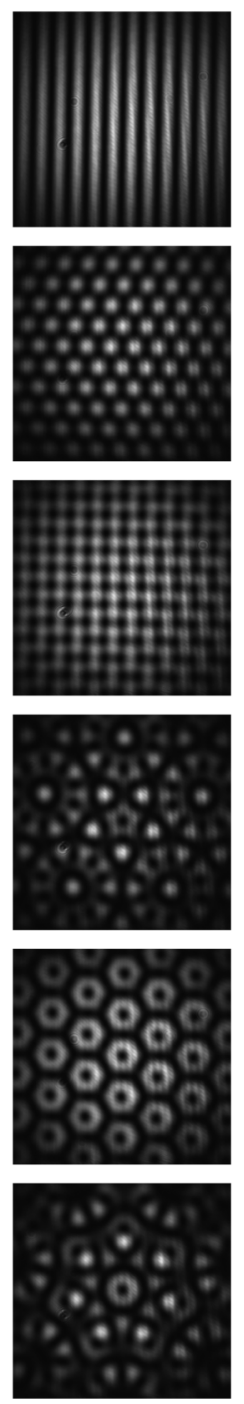

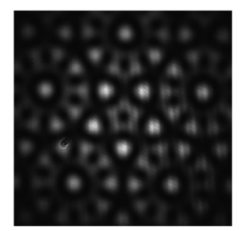

$\mathrm{I}=2$
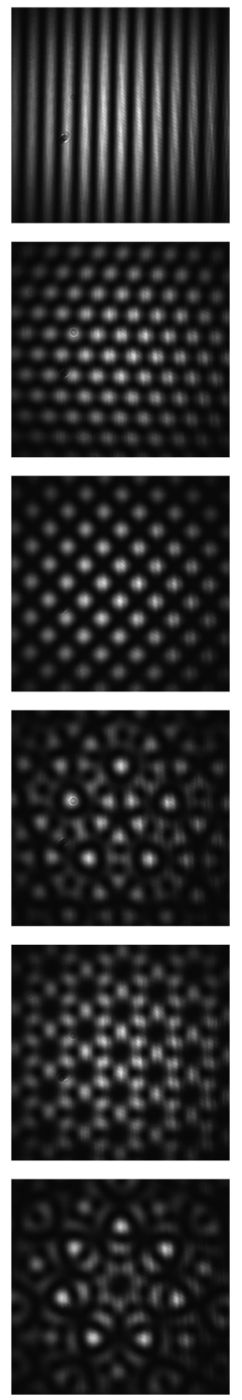

FIG. 5. Measured far-field intensity patterns behind an MPI of $N$ points illuminated by an LG beam with azimuthal mode index $l$. The measured patterns show excellent agreement with the calculated ones (compare Fig. 2).

taken to combine the light, for example, using telescopes and mirrors. This is what is foreseen in the Darwin mission [20]. Darwin is a space-based infrared nulling interferometer that combines the light from four telescope satellites in a central beam combiner satellite. As the telescopes are small with respect to the distance between them, the system operates as a multi-pinhole interferometer. The light from the different telescopes follows paths of equal length to the beam combiner. In fact, the nulling in Darwin is obtained by introducing phase shifts between the telescopes that correspond to a $l=1$ mode, converting an incoming plane wave into a vortex with a null in the center. This is essentially the same as is done in a vortex coronagraph [21], effectively making Darwin a synthetic aperture version of a vortex coronograph. Darwin would be a per- fect detector for astronomical OAM at length scales of hundreds of meters, provided that the different interference patterns can be distinguished. The technique of multipoint interferometry can also be implemented in other existing and future telescope arrays, such as the the VLTI [22], ALMA [23] and KEOPS [24], albeit slightly modified because of the noncircular arrangement of the individual telescopes.

In conclusion, we discussed a new interferometric technique to probe the OAM of light. This technique uses an MPI which makes use of the interferometric combination of the light from a finite number of point measurements. This allows to scan a field for phase singularities and measure their corresponding OAM of light, does not require knowledge of the direction of the beam, and can be scaled to arbitrary dimensions because it samples only a finite number of points in the field. This scalability makes the detection method very useful for systems where the optical vortices are expected to be large, such as in astrophysics. Multi-telescope systems can be used as OAM detectors provided that at least four telescopes are used.

We acknowledge Martin van Exter and Eric Eliel of Leiden University for their contribution to the discussions on this subject.

*j.berkhout@cosine.nl

[1] L. Allen et al., Phys. Rev. A 45, 8185 (1992).

[2] G. Molina-Terriza et al., Nature Phys. 3, 305 (2007).

[3] R. Beth, Phys. Rev. 50, 115 (1936).

[4] H. He et al., Phys. Rev. Lett. 75, 826 (1995).

[5] M.W. Beijersbergen et al., Opt. Commun. 112, 321 (1994).

[6] M. S. S. I. V. Basistiy and M. V. Vasnetsov, Opt. Commun. 119, 604 (1995).

[7] G. Brand, Am. J. Phys. 67, 55 (1999).

[8] N. B. Baranova et al., JETP Lett. 33, 195 (1981).

[9] I. Freund, J. Opt. Soc. Am. A 11, 1644 (1994).

[10] J. F. Nye and M. V. Berry, Proc. R. Soc. A 336, 165 (1974).

[11] M. Harwit, Astrophys. J. 597, 1266 (2003).

[12] B. Thidé et al., Phys. Rev. Lett. 99, 087701 (2007).

[13] J. Leach et al., Phys. Rev. Lett. 88, 257901 (2002).

[14] M. Harris et al., Phys. Rev. A 49, 3119 (1994).

[15] M. Padgett et al., Am. J. Phys. 64, 77 (1996).

[16] T. Young, Philos. Trans. R. Soc. London 12, 387 (1802).

[17] H. I. Sztul and R. R. Alfano, Opt. Lett. 31, 999 (2006).

[18] G. Gbur, T. Visser, and E. Wolf, Opt. Commun. 239, 15 (2004).

[19] C. H. Gan and G. Gbur, Opt. Commun. 280, 249 (2007).

[20] C. S. Cockell et al., arXiv:0805.1873v1 [Astrobiology (to be published)].

[21] G. Foo, D. M. Palacios, and G. A. Swartzlander, Jr., Opt. Lett. 30, 3308 (2005).

[22] URL http://www.eso.org/projects/vlti/.

[23] URL http://www.eso.org/sci/facilities/alma/.

[24] F. Vakili et al., Proc. SPIE Int. Soc. Opt. Eng. 5491, 1580 (2004). 